\title{
Experimental results from face and lateral fine grinding of fused silica and BK7 using metal and resin bonded tools
}

\author{
Christian Schulze ${ }^{1, *}$, Sebastian Henkel $^{1}$, and Jens Bliedtner ${ }^{1}$ \\ ${ }^{1}$ Ernst-Abbe University of Applied Sciences Jena, Carl-Zeiss-Promenade 2, 07745 Jena, Germany
}

\begin{abstract}
The experimental setup shall compare face grinding with lateral grinding in a single processing step using fused silica and BK7 as materials. Resin and metal bonded tools are used in face and lateral grinding strategies. The paper presents the results in order to deepen the knowledge about those grinding technologies and to further improve the properties of fabricated components. Furthermore, the results confirm, that ultrafine grinding is a technology, which can be used to process inorganic non-metallic materials with high quality surfaces with a low roughness and high flatness in a low processing time.
\end{abstract}

\section{Experimental setup}

A 5-axis CNC machine from company SAUER (DMG) with integrated ultrasonic assistance was used to conduct the experiments on both materials, fused silica as well as BK7. The experimental setup with an optimized sample geometry was already described in a previous paper of last year's EOSAM.[1] Two diamond tools with different bonds are used to compare face grinding with lateral grinding in a single processing step. The tool diameter is $14 \mathrm{~mm}$ with an average grain size of $16 \mu \mathrm{m}$ (D16, C50). The compared sample areas are either face grinded/lateral grinded only or processed with both technologies combined.

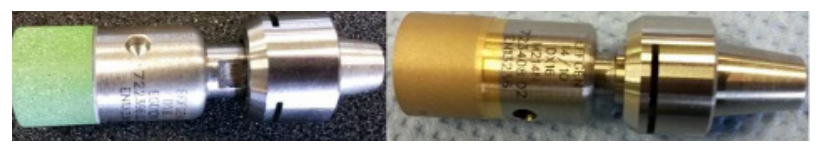

Fig. 1. Resin bonded tool(left) and metal bonded tool (right)

The process influence of the following parameters is investigated:

- 2 materials: fused silica, BK7

- 2 diamond tools: metal bond, resin bond

- 3 values of cutting depth ap

- 2 values of cutting width ae

- $\quad$ state of ultrasonic assistance (on, off)

Table 1. Grinding parameters

ultra fine grinding
\begin{tabular}{|r|r|r|r|r|}
\hline$v_{f}[\mathrm{~mm} / \mathrm{min}]$ & $v_{c}[\mathrm{~m} / \mathrm{s}]$ & $\mathrm{n}\left[\mathrm{min}^{-1}\right]$ & $a_{\mathrm{e}}[\mathrm{mm}]$ & $\mathrm{a}_{\mathrm{p}}[\mathrm{mm}+\mu \mathrm{m}]$ \\
\hline 60 & 22 & 30012 & 0.003 & $2+3$ \\
\hline & & 0.011 & $4+7$ \\
\hline
\end{tabular}

\footnotetext{
* Corresponding author: christian.schulze@eah-jena.de
}

The depth of cut is varied in three steps to gain knowledge about the influence of removal volume in regards to the cutting width, while regarding the special step like sample geometry. The stated number in $\mathrm{mm}$ represents the infeed of one lane, the number stated in $\mu \mathrm{m}$ represents the cutting depth for the last lane, where the tool is in contact with the material again for face grinding. The examined parameters are the roughness and waviness. Furthermore, these parameters are distinguished by their direction of measurement according to the direction of the feed rate (parallel, vertical) and the position of the measurement (single/combined processing and horizontal/vertical surface).

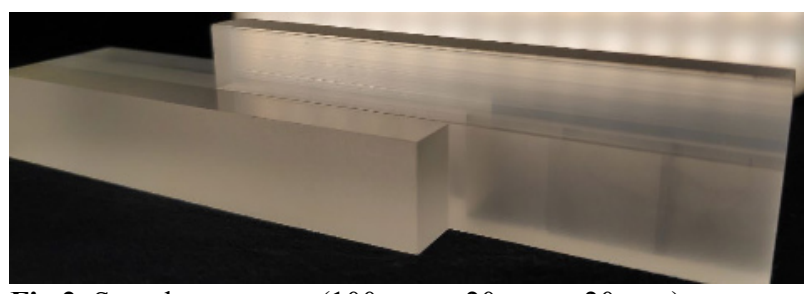

Fig.2. Sample geometry $(100 \mathrm{~mm} \times 20 \mathrm{~mm} \times 20 \mathrm{~mm})$

\section{Parameter significance}

After conducting the experiments and analysing the results, it shows that the parameter with the highest significance is the bond type of the tool, followed by the usage of ultrasonic assistance. Only a shallow level of significance could be recognized for the cut of depth. The cut of width had almost no influence on the results.

Except for the vertical measurement direction on the vertical surface, there are no significant deviations for the measurement directions. There are no remarkable differences in processing the materials using a face/lateral grinding only strategy in comparison to a combined grinding strategy. 


\section{Results}

As shown in fig. 3 and 4, a higher surface quality can be achieved processing fused silica compared to BK7 using identical grinding conditions. Furthermore, using the resin bonded tool for both face and lateral grinding achieves a superior surface quality compared to the metal bonded tool. For lateral grinding the gap of attainable surface quality between both tools, decreases. Especially when processing BK7.

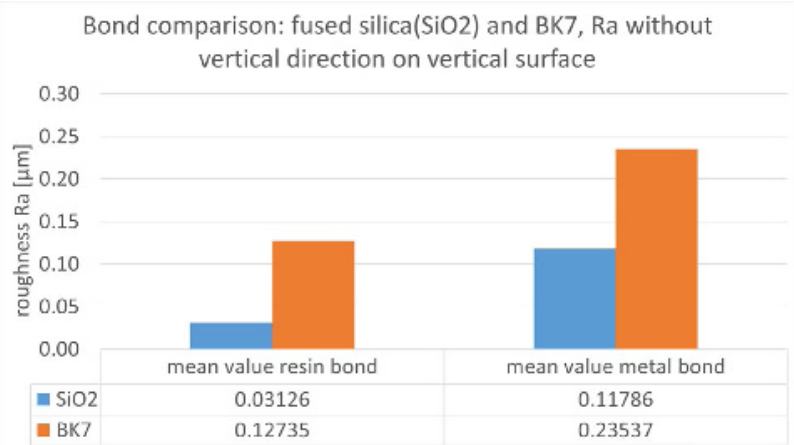

Fig. 3. Comparison: bond, mean value

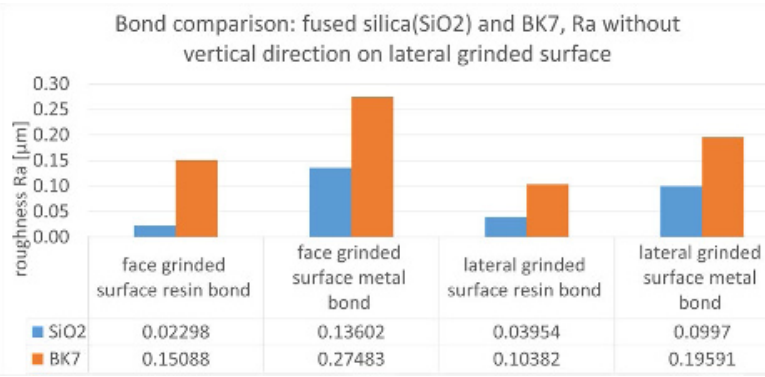

Fig. 4. Comparison: bond, face/lateral grinded surface

Fig. 5. represents the degradation of the surface quality when using ultrasonic assistance. While the mean value of roughness almost doubles for fused silica, it is increased by about $20 \%$ for BK7. Even though the overall mean value of the experiments increases, the ultrasonic assistance shows slight improvements of about $5 \%$ to $10 \%$ for the quality of the vertical surface for lateral grinding.

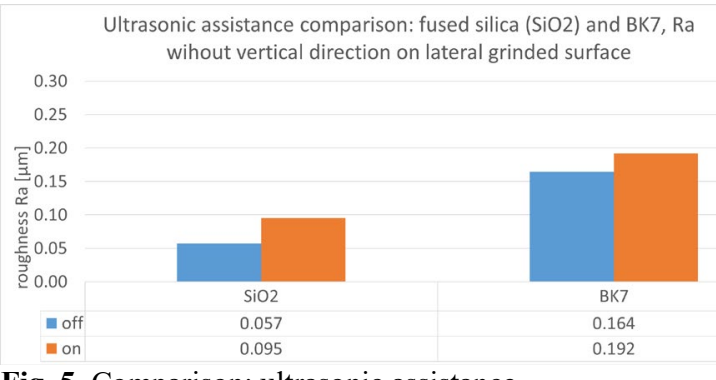

Fig. 5. Comparison: ultrasonic assistance

The achievable flatness lies within a range of about 0.4 $\mu \mathrm{m}$ to $0.8 \mu \mathrm{m}$ without remarkable peculiarities.

The vertical measurement direction of the lateral grinded surface is not taken into consideration for the mean values, since its values are 10 to 20 times higher compared to the other measurement directions. A possible explanation can be found when looking at the footprint the tools produce on the surface with a white light interferometer (WLI). While face grinding creates a smooth surface with circular structures with depths around $1 \mu \mathrm{m}$ (fig. 6), lateral grinding generates a groove profile due to its kinematics, as seen in fig. 7. Those groove profiles can have a total height of more than $10 \mu \mathrm{m}$. In general, the vertical direction of the vertical surfaces shows a deviant behaviour and needs to be further examined.

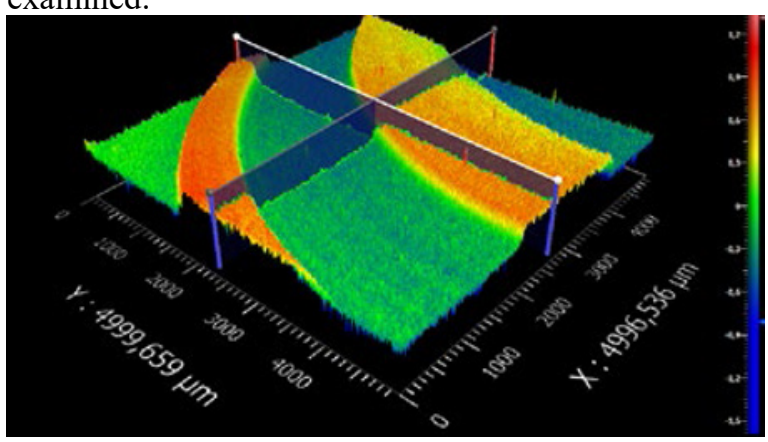

Fig.6. WLI: footprint tool: face grinding

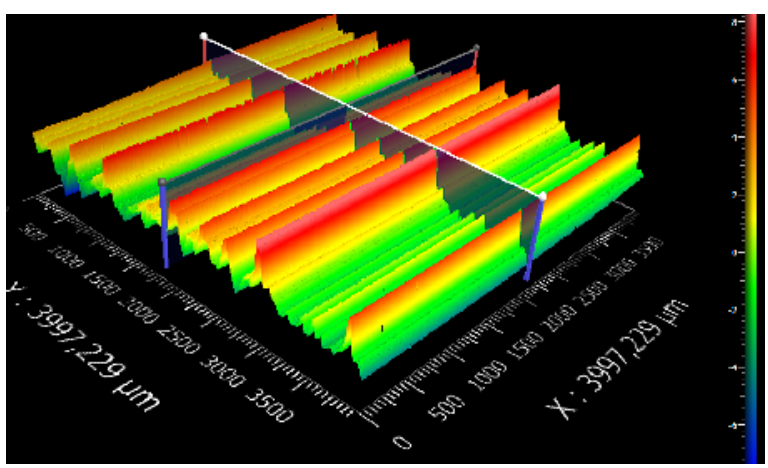

Fig. 7. WLI: footprint tool: lateral grinding

\section{Conclusion}

Overall the conducted experiments have shown, that the used technology is able to create high quality surfaces with a low roughness and high flatness.

The resin bond achieved a better surface quality in comparison to the metal bonded tool using identical tool geometries and grain sizes. Most of the time the usage of ultrasonic assistance resulted in a degradation of the surface quality except for some cases of the vertical surface. Generally speaking a better surface quality could be achieved for processing fused silica in comparison to BK7. Further investigations, especially for lateral grinding are needed to deepen the knowledge and improve the properties of fabricated components.

The authors gratefully acknowledge financial support by the German Federal Ministry of Education and Research, Funding reference: 13FH003IA6 "OpTec 4.0".

\section{References}

1. C. Schulze, S. Henkel, J. Bliedtner: Ultra-fine grinding of inorganic non-metallic materials using various types of bonds and processing strategies. EOSAM 2020, EPJ Web of Conferences Vol.238, article number 03011. (2020) https://doi.org/10.1051/epjconf/202023803011 